\title{
Pilot study to develop telehealth tinnitus management for persons with and without traumatic brain injury
}

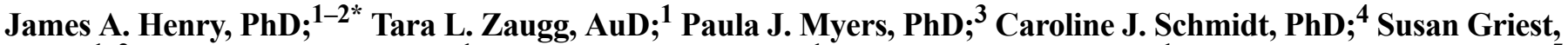 \\ MPH; ${ }^{1-2}$ Marcia W. Legro, PhD; ${ }^{1}$ Christine Kaelin, MBA; ${ }^{1}$ Emily J. Thielman, MS; ${ }^{1}$ Daniel M. Storzbach, PhD; ${ }^{5}$ \\ Garnett P. McMillan, PhD; ${ }^{1}$ Kathleen F. Carlson, PhD $^{6}$ \\ ${ }^{1}$ Department of Veterans Affairs (VA) Rehabilitation Research and Development National Center for Rehabilitative Audi- \\ tory Research, VA Medical Center, Portland, OR; ${ }^{2}$ Department of Otolaryngology/Head and Neck Surgery, Oregon Health \\ \& Science University (OHSU), Portland, OR, ${ }^{3}$ James A. Haley Veterans' Hospital, Tampa, FL; ${ }^{4}$ VA Connecticut Healthcare \\ System, West Haven, CT; and Department of Psychiatry, Yale School of Medicine, New Haven, CT; ${ }^{5}$ Neuropsychology \\ Clinic, VA Medical Center, Portland, OR; and Departments of Psychiatry and Neurology, OHSU, Portland, OR; ${ }^{6}$ VA Health \\ Services Research and Development Portland Center for the Study of Chronic, Comorbid Mental and Physical Disorders, \\ VA Medical Center, Portland, OR; and Department of Public Health and Preventive Medicine, OHSU, Portland, OR
}

\begin{abstract}
Tinnitus, or "ringing in the ears," affects 10\%-15\% of adults; cases can be problematic and require lifelong management. Many people who have experienced traumatic brain injury (TBI) also experience tinnitus. We developed Progressive Tinnitus Management (PTM), which uses education and counseling to help patients learn how to self-manage their reactions to tinnitus. We adapted PTM by delivering the intervention via telephone and by adding cognitive-behavioral therapy. A pilot study was conducted to evaluate the feasibility and potential efficacy of this approach for individuals with and without TBI. Participants with clinically significant tinnitus were recruited into three groups: probable symptomatic mild TBI $(n=15)$, moderate to severe TBI $(n=9)$, and no symptomatic TBI $(n=12)$. Participants received telephone counseling (six sessions over 6 months) by an audiologist and a psychologist. Questionnaires were completed at baseline, 12 weeks, and 24 weeks. All groups showed trends reflecting improvement in self-perceived functional limitations due to tinnitus. A follow-up randomized clinical study is underway.
\end{abstract}

Key words: blast injury, clinical trial, cognitive-behavioral therapy, education, hearing disorders, military Veterans, rehabilitation, telehealth, tinnitus, traumatic brain injury.

\begin{abstract}
Abbreviations: $\mathrm{CBT}=$ cognitive-behavioral therapy, ESS $=$ Epworth Sleepiness Scale, HADS = Hospital Anxiety and Depression Scale, ICF = informed consent form, IED = improvised explosive device, IRB = institutional review board, LOC = loss of consciousness, $\mathrm{m}$-sTBI $=$ moderate to severe traumatic brain injury (group), mTBI = probable symptomatic mild traumatic brain injury (group), NCRAR = National Center for Rehabilitative Auditory Research, noTBI = no symptomatic traumatic brain injury (group), OIF = Operation Iraqi Freedom, PC-PTSD = Primary Care Posttraumatic Stress Disorder screening tool, PTM = Progressive Tinnitus Management, PTSD = posttraumatic stress disorder, PVAMC $=$ Portland Department of Veterans Affairs Medical Center, SD = standard deviation, TBI = traumatic brain injury, THI = Tinnitus Handicap Inventory, THS = Tinnitus and Hearing Survey, VA = Department of Veterans Affairs, VACHS = Department of Veterans Affairs Connecticut Healthcare System, VHA = Veterans Health Administration.

*Address all correspondence to James A. Henry, PhD; VA Medical Center (NCRAR), PO Box 1034, Portland, OR 97207; 503-220-8262, ext 57466; fax: 503-402-2955.

Email: james.henry@va.gov

http://dx.doi.org/10.1682/JRRD.2010.07.0125
\end{abstract}




\section{INTRODUCTION}

Traumatic brain injury (TBI), defined generally as a traumatically induced structural injury or physiological disruption of brain function resulting from external forces to the head, is a leading cause of death and disability in the United States, with an estimated incidence of 1.4 million persons per year-excluding war [1-2]. Sources of TBI include motor vehicle crashes, assaults, contact sports, falls, firearms, and blasts/explosions. TBI is often categorized as mild, moderate, or severe according to the duration of loss of consciousness (LOC) and posttraumatic amnesia. Mild TBI, synonymous with concussion, is the most frequently occurring category, comprising approximately 85 percent of all TBI episodes [3]. Mild TBI has been clinically operationalized as a nonpenetrating force to the head that results in (1) confusion or disorientation, LOC for $30 \mathrm{~min}$ or less, posttraumatic amnesia for less than $24 \mathrm{~h}$, and/or other transient neurological abnormalities such as focal signs, seizure, and intracranial lesion not requiring surgery; and (2) a Glasgow Coma Scale score of 13 to 15 after 30 min postinjury or later upon presentation for healthcare [4]. TBI of all severity levels can cause substantial long-term functional disability among survivors [1]. While symptoms of mild TBI will often resolve given time and no subsequent insults to the head $[3,5]$, a minority of individuals $(\sim 15 \%)$ may experience long-term cognitive, emotional, and physical symptoms after the injury event [3].

TBI is a major problem among returning Operation Iraqi Freedom (OIF) and Operation Enduring Freedom Veterans, with reports suggesting approximately 20 percent having experienced at least a mild TBI [6]. Combatrelated TBI in this new generation of Veterans is most frequently associated with blasts caused by improvised explosive devices (IEDs) or other sources [7]. Like civilian TBI, the majority of combat-related TBIs are deemed to be mild in severity. However, reports have suggested that symptoms associated with mild TBI may be occurring in a larger proportion of Veterans than what has previously been observed in the civilian population [8]. This may be a result of Veterans having incurred multiple TBI events during deployments, greater destructive forces associated with IEDs and other combat blasts, or concomitant mental health problems associated with deployments (e.g., depression, posttraumatic stress disorder [PTSD]).

In both civilians and Veterans, TBI is often associated with concurrent trauma to the auditory system. Clinical and epidemiological studies confirm that TBI is strongly associated with tinnitus [9]. In the past decade, it has become evident that blast-related TBI may be more strongly associated with tinnitus than non-blast-related TBI. For example, in a subset of blast-injured patients seen at Walter Reed Army Medical Center (2003-2005), 49 percent reported tinnitus [10]. Also, 38 percent of inpatients with blast injury at the Palo Alto Department of Veterans Affairs (VA) Polytrauma Rehabilitation Center complained of tinnitus [11]. Tinnitus can occur not only as a symptom of TBI and side effect of medications commonly used to treat cognitive, emotional, and pain problems associated with TBI, but can also be a direct consequence of the event causing TBI, as in the case of blasts. Chandler and Edmond note that tinnitus is a common but often underreported auditory dysfunction with blast-related injury [12]. Cave et al. stated, "Tinnitus can be particularly prevalent for patients who suffer from it secondary to blast injury to their ears, because of the sudden onset of tinnitus in the case of blast injury, instead of the gradual onset of tinnitus developing slowly with progressive hearing loss.... The deleterious effect of tinnitus is highly variable, and medical treatment facilities need to provide subject matter experts who can evaluate the significance of this problem for patients and provide treatment/management as necessary.” [10, p. 729]

Our research has focused on developing effective, evidence-based methods of tinnitus management for Veterans. These efforts led to the development of Progressive Tinnitus Management (PTM), a program involving patient educational counseling that assists patients in learning how to self-manage their reactions to tinnitus [13]. For the present study, we were interested in exploring the feasibility of adapting PTM to address the tinnitus management needs of Veterans and military personnel with TBI by providing the educational counseling via home-based telehealth (telephone counseling).

Telehealth (or telemedicine) encompasses a range of clinical services performed when distance separates provider and patient. These services include medical diagnosis, health monitoring, and therapy. Following passage of the Comprehensive Telehealth Act by Congress in 1997, telehealth services have increasingly been provided in rural or isolated communities [14]. In addition to overcoming geographic barriers, telehealth generally increases accessibility and efficiency of clinical services [15]. Implementing and researching telehealth services is a major initiative for the Veterans Health Administration (VHA) [16]. 
Home-based telehealth refers to the provision of healthcare services remotely to patients in their home environment. Home-based telehealth is an appropriate option for chronic conditions when in-person appointments are unnecessary and the methodology has been demonstrated to be effective [17]. Bell et al. conducted a randomized clinical trial to measure the effectiveness of a scheduled telephone intervention offering counseling and education to people with TBI on behavioral outcomes [18]. Scheduled telephone counseling and education resulted in improved overall outcome, particularly for functional status and quality of well-being, when compared with usual outpatient care. Telephone counseling was described as showing promise as a low-cost, widely available rehabilitation intervention for TBI. Bell et al.'s study lends support to the idea of conducting home-based tinnitus management for Veterans and military personnel with TBI.

The objective of this pilot study was to develop and test the feasibility and potential efficacy of a telehealth tinnitus-management approach for Veterans and military personnel with TBI. The telephone intervention was based on the educational counseling that is used with PTM, with the addition of components of cognitive-behavioral therapy (CBT). CBT is an evidence-based psychotherapy commonly used for pain, depression, anxiety, and tinnitus that also can be effective in individuals with a history of TBI, particularly mild TBI [19-21].

\section{METHODS}

\section{Overview}

This pilot study was a three-group nonrandomized observational study. All participants experienced "clinically significant" tinnitus (i.e., tinnitus that disrupts at least one important life activity and/or causes emotional reactions, resulting in a noticeable reduction in quality of life). One group had probable symptomatic mild TBI (mTBI) $(n=15)$, the second group had moderate to severe TBI $(\mathrm{m}-\mathrm{sTBI})(n=9)$, and the third group had no symptomatic TBI (noTBI) $(n=12)$. The study design specified approximately 10 participants in each group. The purpose was to determine whether telehealth intervention for tinnitus was feasible and efficacious for each of the three groups. Military and VA medical centers were contacted to recruit individuals with clinically significant tinnitus - both with and without a history of TBI. Interested persons called a tollfree number to inquire about the study. The research assistant conducted initial screening for tinnitus and TBI and mailed materials to eligible candidates. The research coordinator assessed capacity to provide informed consent, obtained informed consent as appropriate, and set up the initial telephone counseling appointments. An audiologist and a psychologist conducted these appointments at (approximately) 5, 6, 7, 8, 12, and $24 \mathrm{wk}$. The intervention consisted primarily of efforts to facilitate the therapeutic use of sound and CBT techniques to effectively manage reactions to tinnitus. Outcomes assessment was conducted using the Tinnitus Handicap Inventory (THI) at baseline and at 12 and $24 \mathrm{wk}$ following baseline.

\section{Progressive Tinnitus Management}

PTM is a hierarchical method of providing clinical services for patients who complain of tinnitus. The method has been described in detail [13] and is described briefly here. The hierarchy of clinical services includes five levels. Level 1 Triage addresses patients at the initial point of contact [22]. Level 1 consists of guidelines for referring patients who complain of tinnitus to the appropriate clinical services. Unless the patient has urgent medical or mental health concerns, the initial services should involve a hearing assessment and brief assessment of tinnitus impact. These initial services comprise the level 2 Audiologic Evaluation [23]. During the level 2 evaluation, the brief assessment of tinnitus impact determines whether tinnitus-specific services are appropriate. If so, patients normally are advised to attend level 3 Group Education workshops.

As originally developed, the level 3 workshops consisted of two group sessions facilitated by an audiologist [24]. Sessions were $1.5 \mathrm{~h}$ in length and separated by $2 \mathrm{wk}$. The structured educational curriculum focused on teaching patients how to develop action plans for using sound in specific ways to address situations when the tinnitus was most bothersome. The education was designed to be patient-centered and to lead to self-efficacy in managing reactions to tinnitus.

Patients who need further help after attending the level 3 workshops are advised to undergo a level 4 Interdisciplinary Evaluation [13]. Level 4 services are required by relatively few patients and involve primarily in-depth interviews by an audiologist and psychologist, who attempt to determine why the tinnitus continues to be a problem. If further intervention is deemed necessary, then patients are advised to receive level 5 Individualized Support, which normally involves up to 6 mo of one-on-one counseling by the audiologist and/or the psychologist. 


\section{Modification of Progressive Tinnitus Management for Use With This Study}

The study team included (but was not limited to) a neuropsychologist (DMS) with expertise in TBI, an audiologist (PJM) with expertise in TBI and patient health education, and a research psychologist (CJS) with expertise in using CBT for tinnitus management. These collaborators assisted in modifying the PTM protocol so that it was appropriate for telephone-based administration to TBIaffected persons. In general, PTM was condensed to conducting assessments using questionnaires and interviews and performing telephone counseling as the intervention. Specific changes to the PTM protocol included-

1. The study participants underwent a cognitive screen to determine their ability to comprehend the counseling information and to follow through with the management recommendations.

2. Guidelines were developed to assign participants to different categories of TBI.

3. Counseling materials were oriented to address low health literacy.

4. CBT and sleep hygiene tips were added to the protocol to help the participants learn coping techniques that were not addressed by the earlier version of the PTM counseling.

\section{Cognitive-Behavioral Therapy}

Before this pilot study, psychological concerns associated with tinnitus were not specifically addressed by the PTM intervention. Some people distressed by their tinnitus report symptoms of depression and anxiety, such as sleep disturbance, difficulty concentrating, fatigue, irritability, and even suicidal ideation [25-27]. Psychological techniques had already been developed and used with patients who experience tinnitus. Multiple trials support CBT as an effective psychological method for managing tinnitus [20]. Thus, CBT was incorporated into the PTM method and was expected to be useful for patients both with and without TBI.

CBT is a type of psychotherapy that targets specific thoughts, core beliefs, and negative appraisals of situations that are unconstructive (and may cause distress) while providing tools for implementing more adaptive behavioral and cognitive modifications [28-29]. Martinez Devesa et al. conducted a meta-analysis of six randomized controlled studies (285 participants) of CBT for tinnitus [20]. They found significant improvement in quality of life (decrease of global tinnitus severity) for those receiv- ing CBT compared with those who did not receive CBT. CBT is an evidence-based and appropriate addition to PTM to address the psychological components of tinnitus distress that are so common with Veterans.

CBT often involves six to eight sessions to cover all components of the program. Only two coping techniques were introduced with this trial of PTM to enable all the telephone counseling to be covered in a reasonable number of sessions. The components used in this study included training in behavioral modification (stress management via relaxation techniques), as well as cognitive restructuring (step-by-step examination of changing thoughts to change how people feel). These particular components were selected because they have been identified in studies to be beneficial for patients who have tinnitus [30-31].

\section{Self-Help Workbook}

We developed a patient self-help workbook for use with our clinic-based PTM program [32]. The workbook was designed so that the information would be comprehensible to people with low health literacy [33]. The primary purpose of the workbook was to impart useful, actionable information that helps patients make good decisions about their tinnitus healthcare. The focus of the workbook was to facilitate the development of individualized "sound management plans" (using the Sound Plan Worksheet in Appendix 1, available online only). Specifically, instructions were provided for how to use sound to manage tinnitus in situations when tinnitus is bothersome. Because tinnitus is a chronic and permanent condition, an additional objective of the workbook was to convey information that is important to prevent exacerbation of tinnitus and hearing loss, to enhance quality of life, and to reduce complications and/or problems related to coping with the condition.

The addition of CBT to the clinic-based PTM protocol required expansion of the self-help workbook, so we developed a second edition of the workbook that was sent to all participants at the time of their enrollment [34]. The workbook was revised with assistance from the psychologist (CJS) to incorporate cognitive and behavioral coping skills for patients. The CBT information led to development of a worksheet (Changing Thoughts and Feelings Worksheet in Appendix 2, available online only) to develop an action plan for implementing specific tasks relevant to managing reactions to tinnitus. This new workbook also contains videos that supplement the written material: (1) an interactive video that guides patients through material in the workbook about therapeutic use of sound (material that normally is 
presented in PTM group education workshops) (Video 1), and (2) demonstrations of relaxation training exercises including deep breathing and imagery (Video 2).

\section{Intervention Counselors}

All the telephone interventions were conducted by the research audiologist (TLZ) and research psychologist (CJS). The audiologist was located at the National Center for Rehabilitative Auditory Research (NCRAR), which is hosted by the Portland VA Medical Center (PVAMC) in Portland, Oregon. The psychologist was located at the VA Connecticut Healthcare System (VACHS) in West Haven, Connecticut. The audiologist participated in the development of the patient education that originally was developed for PTM and had experience using it. The psychologist was experienced in providing CBT to patients with tinnitus. Both the audiologist and psychologist completed online VA-endorsed training courses that provide information about people with TBI and PTSD.

\section{Database}

Three centralized databases were used:

1. Microsoft Outlook (Microsoft Corp; Redmond, Washington) served as a schedule management tool, enabling the research assistant, research coordinator, and intervention counselors to effectively schedule appointments and reminders.

2. A Microsoft Access (Microsoft Corp) relational database was developed to collect screening and outcome data. Whenever the research assistant, research coordinator, or an intervention counselor talked to a candidate or participant on the telephone, the database was opened to the person's record. Information could be entered into the database in real time as the individual provided information and responded to questions.

3. In addition, outcome data were entered from questionnaires that were completed and returned by participants. In lieu of a paper chart, the intervention counselors (audiologist and psychologist) had access to the completed outcome questionnaires that were scanned into a database that both could view.

\section{Recruitment and Enrollment}

All procedures for recruitment, informed consent, and conduct of this study adhered to the requirements of the institutional review boards (IRBs) at the PVAMC and VACHS for protection of human research participants. Participants included only Veterans and military person- nel who were informed of the study via a recruitment flyer that was sent to VA and military audiologists.

Communications with candidates and participants were made via telephone (using the PVAMC and VACHS tollfree numbers for inbound calls). For documentation and security purposes, all deliveries of the informed consent form (ICF) and questionnaires were sent to/from participants via express carrier. (Figure 1 contains a flowchart showing screening and enrollment numbers.)

\section{Inclusion/Exclusion Criteria}

Study candidacy was determined by both the research assistant and research coordinator. Inclusion criteria included (1) "clinically significant" tinnitus as determined by a minimum total score of 3 on section A of the Tinnitus and Hearing Survey (THS) (Appendix 3, available online only), (2) demonstrated understanding of the requirements of the study (based on adequate responses to the research coordinator's questions that assessed capacity-to-consent), and (3) motivation and capability to participate (including ability to communicate over the telephone in English). Callers who did not meet all these criteria were excluded from study participation.

\section{Tinnitus and Hearing Survey}

It is common for patients to erroneously blame hearing problems on tinnitus [35]. Because the telehealth intervention was designed to help only with tinnitus problems, and not with hearing problems, it was necessary to distinguish these two problems to determine candidacy for the study. Use of the THS (Appendix 3) enables rapid assessment of the effects of hearing loss separate from the effects of tinnitus $[23,32]$. The statements in section A address tinnitus-specific problems not related to hearing difficulties. Section B contains statements that focus on common hearing problems. All the problems in section A are problems that can be addressed with intervention specific to tinnitus that would be available through the telehealth program. Addressing the problems in section B would need to begin with an audiologic evaluation and could not be managed through the telehealth program. Section C contains two statements that are used to screen for a sound tolerance problem (also known as "hyperacusis"). The THS was used as an eligibility screening tool and as part of a secondary outcomes assessment (data not reported in this article). It also could be used as a clinical tool by the research audiologist during the intervention appointments. 


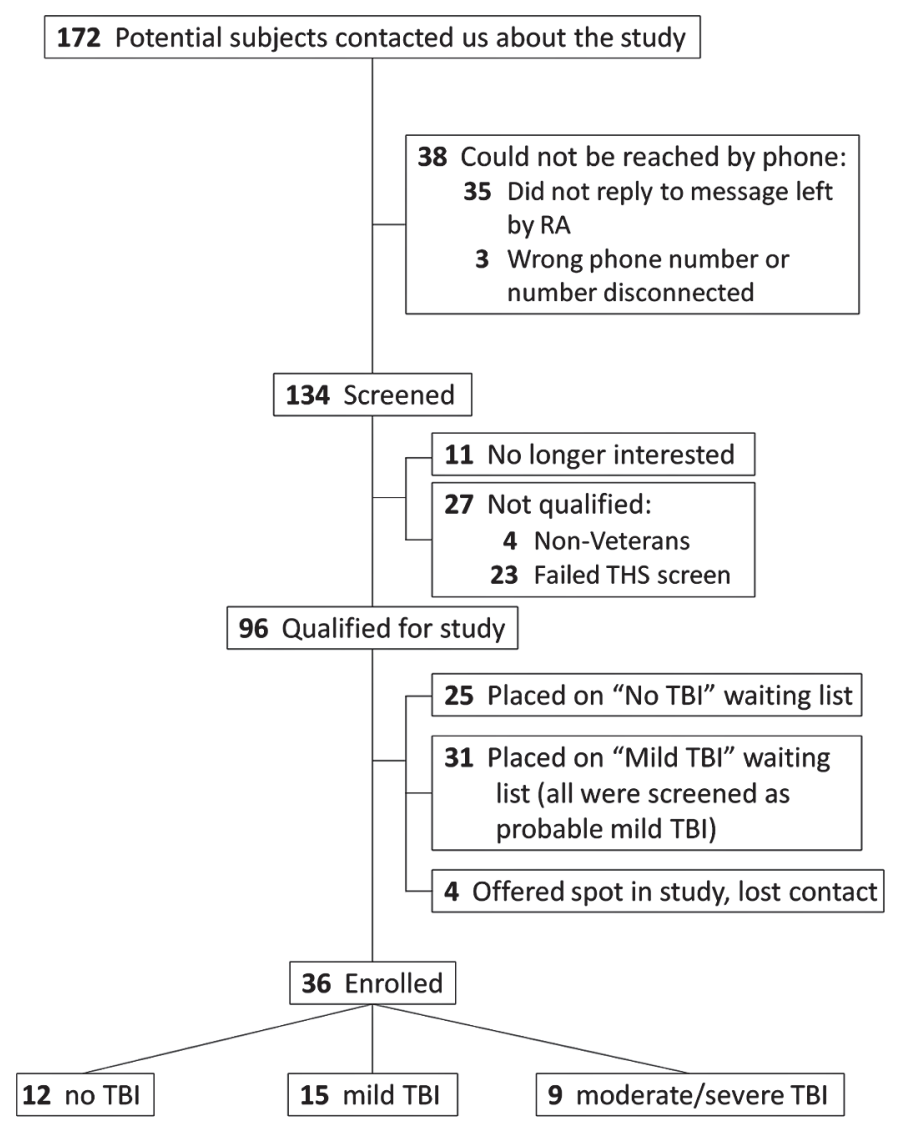

Figure 1.

Number of callers expressing interest in pilot study with regard to how many were recruited, screened, enrolled, and assigned to three study groups. As of November 2009, 172 potential candidates had contacted research assistant (RA) to express interest in study. Of these, 96 were screened and found to be qualified candidates. Of qualified candidates, 36 were enrolled in study (rest were placed on waiting list or lost contact). Note that those placed on waiting list were informed that we would attempt to renew study, but that renewal required approval for continued funding. $\mathrm{TBI}=$ traumatic brain injury, THS = Tinnitus and Hearing Survey.

\section{Initial Screening}

For initial screening, the research assistant (1) explained the study, (2) administered the THS, and (3) assessed probable symptomatic TBI using a modified version of the VA TBI Screen (Appendix 4, available online only). Callers who responded that they already had been diagnosed with TBI or who endorsed all four sections of the TBI Screen were considered to have probable symptomatic TBI. However, confirmation of TBI history and symptoms and assign- ment to TBI categories took place during the assessment appointment with the psychologist.

Qualified candidates were sent the self-help workbook, ICF, and written baseline questionnaires (Table 1). The baseline questionnaires included (1) Tinnitus Questionnaire, (2) Hospital Anxiety and Depression Scale (HADS), (3) THI, (4) Primary Care PTSD screening tool (PCPTSD), and (5) Epworth Sleepiness Scale (ESS). The HADS, PC-PTSD, and ESS were administered to screen for anxiety and depression, PTSD, and sleep deprivation, respectively. Each of these conditions can compromise efforts to manage tinnitus, so evaluating the participants for these potential comorbidities was essential. The HADS, PC-PTSD, and ESS were selected because of their validated effectiveness for screening purposes. Completed forms for all three of these scales were scanned in the scanned-questionnaires database on receipt so as to be available for the psychologist to review and discuss with the participant during telephone appointments. Participants who screened positive for any of these disorders were encouraged to contact their primary care physicians for referral to appropriate providers.

\section{Traumatic Brain Injury Screen}

Participants were assigned to one of three TBI categories: mTBI, m-sTBI, and noTBI. We highlight that individuals assigned to the mTBI category were those with probable symptomatic mild TBI; individuals with a history of mild TBI but no current symptoms that could be attributable to a TBI were assigned to the noTBI category. Participants with moderate or severe TBI were combined into a separate category; this method was necessary given our relatively low $N$ for this study but also was justified given that mild TBI has been argued to be

Table 1.

Schedule of questionnaire administration.

\begin{tabular}{lcccc}
\hline \multicolumn{1}{c}{ Questionnaire } & Screen & Baseline & $\begin{array}{c}\mathbf{1 2} \\
\mathbf{w k}\end{array}$ & $\begin{array}{c}\mathbf{2 4} \\
\mathbf{w k}\end{array}$ \\
\hline Tinnitus and Hearing Survey & $\mathrm{X}$ & - & $\mathrm{X}$ & $\mathrm{X}$ \\
TBI Screen & $\mathrm{X}$ & - & - & - \\
Tinnitus Questionnaire & - & $\mathrm{X}$ & - & - \\
Hospital Anxiety \& Depression Scale & - & $\mathrm{X}$ & - & - \\
Tinnitus Handicap Inventory & - & $\mathrm{X}$ & $\mathrm{X}$ & $\mathrm{X}$ \\
Primary Care PTSD Screening Tool & - & $\mathrm{X}$ & - & - \\
Epworth Sleepiness Scale & - & $\mathrm{X}$ & - & - \\
\hline PTSD = postraumatic stress disorder, TBI & traumatic brain injury.
\end{tabular}

PTSD $=$ posttraumatic stress disorder, TBI = traumatic brain injury. 
distinct from moderate and severe TBI rather than on one continuum of cognitive, emotional, and physical severity $[3,5]$. For example, outcomes of moderate and severe TBI tend to follow a gradient of severity predicted by acute injury characteristics, while outcomes of mild TBI can be varied and unpredictable [3].

Assignment to one of the three TBI categories was based on responses to the VA TBI Screen plus criteria developed in consultation with TBI screening specialists (PJM and DMS). Criteria were adapted from the Veterans Health Initiative's Traumatic Brain Injury: Independent Study Course (http://www.gesturetekhealth.com/pdf/research/ neurological/4.4.2_USA_VA_TBI_Report.pdf; January 2004) and from Iverson et al. [36]. Participants who had not previously been diagnosed with TBI (according to self-report) and did not endorse all four sections of the TBI Screen (Appendix 4) were assigned to the noTBI category. Those who had previously been diagnosed with TBI or who endorsed all four sections of the TBI Screen were assigned to either the mTBI or the m-sTBI category based on the established criteria. For example, one of the key criteria for assigning participants to a TBI severity category is length of time they experienced LOC at the time of the TBI. Those who had LOC for $30 \mathrm{~min}$ or less were assigned to the mTBI category and those who had LOC for more than $30 \mathrm{~min}$ were assigned to the $\mathrm{m}$-sTBI category.

\section{Tinnitus Questionnaire}

This is a general five-page survey that was completed only at baseline. It obtains demographic data as well as information about the participant's tinnitus and its effects, prior clinical services received for tinnitus, hearing difficulties, and prior services received for hearing loss.

\section{Hospital Anxiety and Depression Scale}

Baseline screening for anxiety and depression was done using the HADS [37]. This self-screening questionnaire consists of 14 questions -7 for anxiety and 7 for depression. The HADS has been used extensively in primary care settings [38]. Responses were used by the psychologist to assist in determining the potential need to address anxiety and/or depression.

\section{Tinnitus Handicap Inventory}

The THI provides an index score, ranging from 0 to 100 , with higher scores reflecting greater perceived functional limitations due to tinnitus [39-40]. The THI has been validated for use with patients, and it is one of the most often used and cited tinnitus instruments. A change in the index score of at least 20 points is reported to indicate a clinically significant improvement for individuals at the 95 percent confidence level [41].

\section{Primary Care Posttraumatic Stress Disorder Screening Tool}

The four-question PC-PTSD is used by the Department of Defense and the VA to detect probable PTSD and to initiate appropriate referral [42]. A cutoff score of 3 has been shown to have 76 percent sensitivity and 92 percent specificity for clinical PTSD among OIF soldiers [43].

\section{Epworth Sleepiness Scale}

The ESS was used to screen participants for sleep disorders [44]. The ESS is the most widely used standardized tool for this purpose. Completing the ESS provides an index score that can be compared with normative data. Responses were used by the psychologist and audiologist to assist in determining the potential need to address sleep disorders as part of the intervention or to refer the participant to a sleep disorders clinic.

\section{Consent Appointment}

The consent appointment took place about 1 to $2 \mathrm{wk}$ following initial screening. During this appointment, the research coordinator explained the ICF and assessed the candidate's capacity-to-consent (assessment of capacity-toconsent was required by the IRB and a set of questions was developed to address this requirement). Assessment of capacity-to-consent simultaneously evaluated the candidate's speech comprehension ability. Failure to pass the capacity-to-consent assessment would exclude the candidate from study participation (no candidates were excluded on this basis). If the candidate was qualified, motivated (as determined by expressed interest), and capable of participation, then the research coordinator instructed the candidate about how to sign the ICF, complete the written questionnaires, and return these items in the prepaid and preaddressed envelope that was provided. Upon receipt of the signed ICF and completed questionnaires, the candidate was enrolled into the study and the research coordinator scheduled the assessment appointment with the psychologist.

\section{Assessment Appointment}

During this TBI and mental health history evaluation appointment, the psychologist (1) assessed for any obvious cognitive impairment via a symptom checklist and mental 
status examination, (2) reviewed the completed screening forms that participants completed at home and assessed for mental health conditions as indicated, (3) asked participants to describe their history of TBI(s) and to report relevant medical data so as to affirm the presence and severity of TBI(s) according to the study protocol, and (4) made appropriate referrals if warranted. Results of the TBI evaluation determined the participant's assignment to one of the three TBI categories. If time permitted, the psychologist introduced the use of CBT for tinnitus, the rationale behind stress management for tinnitus, and the relaxation techniques. Participants were invited to begin reading introductory selections from the self-help workbook.

\section{Participants}

A total of 172 individuals called about the study. (See flowchart in Figure 1 that shows screening and enrollment numbers.) Of these, 36 participants ( 32 male and 4 female) were enrolled, including 15 in the mTBI group (mean \pm standard deviation [SD] age $=59.0 \pm 12.5 \mathrm{yr}), 9$ in the $\mathrm{m}$ $\mathrm{sTBI}$ group (mean $\pm \mathrm{SD}$ age $=56.6 \pm 10.8 \mathrm{yr}$ ), and 12 in the noTBI group (mean \pm SD age $=59.5 \pm 10.8$ yr) $($ Table 2$)$. The 36 participants were located in 18 different states.

\section{Intervention Procedures}

The intervention protocol normally involved six telephone appointments - four with the audiologist (TLZ) and two with the psychologist (CJS). (However, as previously mentioned, the assessment appointment with the psychologist was also an intervention appointment if there was time to start the intervention.) The first intervention appointment was scheduled as soon as possible after the assessment appointment - usually within 1-2 wk. The first four intervention appointments were scheduled approximately weekly and alternated between the audiologist and the psychologist. The last two intervention appointments were conducted with the audiologist at about 12 and 24 wk after

Table 2.

Age and sex of study participants.

\begin{tabular}{lrrrrrr}
\hline \multirow{2}{*}{ Group } & \multirow{n}{*}{$\boldsymbol{n}$} & \multicolumn{2}{c}{ Age (yr) } & & \multicolumn{2}{c}{ Sex } \\
\cline { 3 - 4 } & & Mean \pm SD & Range & & Male & Female \\
\hline No TBI & 12 & $59.5 \pm 10.8$ & $35-76$ & & 12 & 0 \\
Mild TBI & 15 & $59.0 \pm 12.5$ & $38-83$ & & 13 & 2 \\
Moderate to Severe TBI & 9 & $56.6 \pm 10.8$ & $45-79$ & & 7 & 2 \\
Total & 36 & $58.6 \pm 11.3$ & $35-83$ & & 32 & 4 \\
\hline SD = standard deviation, TBI = traumatic brain injury. & \\
\hline \hline
\end{tabular}

enrollment. In addition to the specific procedures outlined subsequently, at each of these appointments the intervention counselor started by explaining the format of the appointment, allowed time to answer questions, made referrals to other relevant healthcare providers if warranted, and ensured that the next intervention appointment was scheduled as appropriate.

These sessions were individualized; thus, the information was provided at a pace that was comfortable for each participant. Information was introduced as time permitted. Participants were asked to complete mutually agreed upon "homework" (written activities) between sessions. If participants were unable to demonstrate having understood the concepts from previous sessions, then those concepts were reviewed. Providers also periodically asked participants to summarize the information immediately after it was provided during a session. If a therapist determined that a participant had not understood a concept, then the concept was reviewed. Participants who were struggling to learn concepts or who appeared to be learning the information at a slower pace were offered additional appointments. If participants were interested in additional appointments, then they were scheduled for these appointments as needed until the final study appointment approximately $24 \mathrm{wk}$ after initiation of intervention. Nine participants accepted additional appointments with the study audiologist when offered, and three accepted additional appointments with the study psychologist when offered. These requests generally resulted in 1-2 additional appointments, but one participant required 5 additional appointments with both the psychologist and audiologist. Note that only one participant dropped out of the study, which occurred during the intervention period.

\section{Appointment 1}

Again, this appointment was scheduled about $1 \mathrm{wk}$ after the "assessment" appointment with the psychologist. The audiologist (1) administered the THS (Appendix 3), (2) explained strategies for using sound to manage reactions to tinnitus, and (3) facilitated completing the Sound Plan Worksheet (Appendix 1) that is contained in the selfhelp workbook [34] to develop an individualized plan to manage reactions to tinnitus over the following 2 wk.

\section{Appointment 2}

The psychologist (1) introduced principles of CBT and stress management (or reviewed if already introduced during the assessment appointment); (2) introduced the 
concept of "mindfulness," whereby patients learn to attend to their own thoughts; and (3) facilitated beginning use of the Changing Thoughts and Feelings Worksheet (Appendix 2) that is contained in the self-help workbook [34] to set goals and develop an individualized plan to psychologically manage reactions to tinnitus over the next $2 \mathrm{wk}$.

\section{Appointment 3}

The audiologist (1) reviewed strategies for using sound to manage reactions to tinnitus, (2) discussed the participant's experience carrying out the sound plan developed at the previous telephone call, (3) worked with the participant to refine the sound plan as needed, and (4) helped the participant to identify tinnitus management activities to implement.

\section{Appointment 4}

The psychologist (1) reviewed CBT for tinnitus and stress management, (2) reviewed the mindfulness skill and the Changing Thoughts and Feelings Worksheet (Appendix 2), (3) introduced healthy attitudes and steps for changing negative appraisals to neutral or positive appraisals, (4) developed a long-term plan to implement CBT coping skills to manage reactions to tinnitus, and (5) administered the THS if deemed appropriate.

\section{Appointment 5}

Procedures with the audiologist were the same as for Appointment 3-modified as appropriate.

\section{Appointment 6}

The audiologist conducted this final appointment to complete the study. The audiologist reviewed the protocol, discussed progress made, and answered any questions. The THS was administered by the audiologist for the final time. (Note that the THS was completed by participants on three occasions: baseline, $12 \mathrm{wk}$, and $24 \mathrm{wk}$.)

\section{Data Analysis}

Because this was a pilot study and the number of participants within each of the TBI groups was small $(\leq 15)$, descriptive data are reported with nonparametric statistical tests and effect sizes for the outcome data. For each of the three groups, Wilcoxon signed rank tests (nonparametric equivalent to paired $t$-tests) were performed to determine whether the THI scores differed significantly between baseline and the two follow-up time points (12 and $24 \mathrm{wk}$ ). A Kruskal-Wallis Test (nonparametric equivalent to a oneway analysis of variance) was performed to determine whether the time effect varied across groups. In addition, we calculated effect sizes to standardize the outcome data [45]. To control for type I error, a Bonferroni correction was made and the significant $p$-value was adjusted to 0.008 .

\section{RESULTS}

\section{Baseline Questionnaires}

The baseline questionnaires were used to obtain demographic information; descriptions of the participants' tinnitus; and screening data for PTSD, depression, anxiety, and sleepiness. Responses for selected questions from the baseline questionnaires are shown across the three groups in Table 3, along with the responses for the HADS, ESS, and PC-PTSD screening tool. The results can be summarized as follows.

\section{What is the Location of Your Tinnitus?}

Most participants localized their tinnitus to both ears (66.7\%). However, a larger number of participants in the $\mathrm{m}$-sTBI group localized their tinnitus to inside their head (44.4\%) compared with the mTBI group $(13.3 \%)$ and noTBI group $(8.3 \%)$.

\section{Is Your Tinnitus One Sound or More?}

The majority of participants (58.3\%) perceived their tinnitus as more than one sound while 36 percent perceived it as a single sound and 11 percent were unsure as to the number of sounds. Those with mTBI were more likely to report a single sound (53.3\%) compared with the noTBI group (16.7\%) and m-sTBI group (33.3\%).

\section{How Much of the Time is Tinnitus Present?}

Most participants reported their tinnitus to be present "most of the time" or "always there" (97.1\%). This distribution was similar across all three groups.

\section{How Much of a Problem is Tinnitus?}

Almost all participants considered their tinnitus to be at least a moderate problem. This distribution was similar across all three groups.

\section{Epworth Sleepiness Scale}

A majority of participants scored within the range of not getting enough sleep (9-24 points). Participants with TBI were more likely to fall within this range than those without TBI (noTBI: 36.4\%, mTBI: 57.1\%, m-sTBI: 66.7\%). 
JRRD, Volume 49, Number 7, 2012

Table 3.

Responses to study questions in no traumatic brain injury (TBI), mild TBI, and moderate to severe TBI groups. Data from baseline questionnaires.

\begin{tabular}{|c|c|c|c|c|}
\hline \multirow[b]{2}{*}{ Question/Response Choices } & \multicolumn{4}{|c|}{ Response (No. of Subjects) } \\
\hline & $\begin{array}{c}\text { No } \\
\text { TBI }\end{array}$ & $\begin{array}{c}\text { Mild } \\
\text { TBI }\end{array}$ & $\begin{array}{c}\text { Moderate-Severe } \\
\text { TBI }\end{array}$ & Total \\
\hline \multicolumn{5}{|l|}{ "What is the Location of Your Tinnitus?" } \\
\hline$n$ & 12 & 15 & 9 & 36 \\
\hline Left Ear Only & 1 & 1 & 1 & 3 \\
\hline Right Ear Only & 0 & 1 & 0 & 1 \\
\hline Inside Head & 1 & 2 & 4 & 7 \\
\hline Other & 0 & 0 & 1 & 1 \\
\hline \multicolumn{5}{|l|}{ "Is Your Tinnitus One Sound or More?" } \\
\hline$n$ & 12 & 15 & 9 & 36 \\
\hline 1 Sound & 2 & 8 & 3 & 13 \\
\hline \multicolumn{5}{|c|}{ "How Much of the Time is Your Tinnitus Present?" } \\
\hline$n$ & 12 & 15 & 8 & 35 \\
\hline Some of Time & 0 & 1 & 0 & 1 \\
\hline Most of Time & 3 & 2 & 4 & 9 \\
\hline Always There & 9 & 12 & 4 & 25 \\
\hline \multicolumn{5}{|l|}{ "How Much of a Problem is Your Tinnitus?" } \\
\hline$n$ & 11 & 15 & 9 & 35 \\
\hline Slight Problem & 0 & 1 & 2 & 3 \\
\hline Moderate Problem & 4 & 3 & 3 & 10 \\
\hline \multicolumn{5}{|l|}{ Screen Positive for PTSD? } \\
\hline$n$ & 12 & 15 & 9 & 36 \\
\hline No & 8 & 4 & 2 & 14 \\
\hline Yes & 4 & 11 & 7 & 22 \\
\hline \multicolumn{5}{|l|}{ HADS/Anxiety Scale_-Range 0-21 } \\
\hline$n$ & 12 & 15 & 9 & 36 \\
\hline Normal-Range 0-7 & 5 & 4 & 1 & 10 \\
\hline Borderline Normal-Range $8-10$ & 2 & 0 & 1 & 3 \\
\hline Abnormal—Range 11-21 & 5 & 11 & 7 & 23 \\
\hline \multicolumn{5}{|l|}{ HADS/Depression Scale_-Range 0-21 } \\
\hline$n$ & 12 & 15 & 9 & 36 \\
\hline Normal-Range $0-7$ & 6 & 5 & 2 & 13 \\
\hline Borderline Normal-Range $8-10$ & 2 & 2 & 4 & 8 \\
\hline Abnormal-Range 11-21 & 4 & 8 & 3 & 15 \\
\hline
\end{tabular}




\section{Screen Positive for Posttraumatic Stress Disorder}

More than half the participants $(61.1 \%)$ screened positive for PTSD. Those with TBI were more likely to screen positive than those without TBI (noTBI: $33.3 \%$, mTBI: 73.3\%, m-sTBI: 77.8\%).

\section{Hospital Anxiety and Depression Scale}

Almost two-thirds $(63.9 \%)$ of the participants scored abnormally high (11-21) on the anxiety portion of the HADS. Those with TBI were more likely to have abnormal anxiety scores than those without TBI (noTBI: $41.7 \%$, mTBI: $73.3 \%$, m-sTBI: 77.8\%).

For the depression portion of the HADS, the majority of participants $(58.3 \%)$ scored within the normal $(0-7)$ or borderline normal (8-10) range. Scores for the mTBI group tended to fall in the abnormal range $(53.3 \%)$ more often than for the m-sTBI (33.3\%) and noTBI (33.3\%) groups.

Participants who screened positive for PTSD were more likely to score in the abnormal range for the HADS anxiety scale (90.9\%), HADS depression scale (54.5\%), and ESS (65\%).

Patients were not screened specifically for suicidal ideations. One participant reported current, passive suicidal ideations during the assessment appointment and during several intervention appointments. Initially risk of self-harm was deemed "low" by the psychologist; thus, a safety plan was implemented. However, during a followup call the participant's risk increased and the participant's local VA suicide coordinator was notified, who acted to ensure safety. Two other participants reported past but not recent or current suicidal ideations.

\section{Tinnitus Handicap Inventory}

Overall results of mean THI scores for each TBI group at each outcome point are shown in Figure 2. These mean values are based on the numbers of participants who completed the THI at each outcome point (Table 4). Mean \pm SD THI scores for the noTBI group were $63.7 \pm 21.1$ at baseline, $41.8 \pm 28.8$ at $12 \mathrm{wk}$, and $40.7 \pm 26.9$ at 24 wk. Mean \pm SD THI scores for the mTBI group were $66.2 \pm 22$ at baseline, $53.4 \pm 28.3$ at $12 \mathrm{wk}$, and $58.6 \pm 29.0$ at $24 \mathrm{wk}$. Mean \pm SD THI scores for the m-sTBI group were $55.1 \pm 22.4$ at baseline, $34.0 \pm$ 24.5 at $12 \mathrm{wk}$, and $36.3 \pm 25.6$ at $24 \mathrm{wk}$.

A nonparametric test (Kruskal-Wallis) determined that the baseline mean values were not significantly different between groups $(p>0.05)$. Wilcoxon signed rank test was used to evaluate changes in THI scores within each group. Data for the baseline to $12 \mathrm{wk}$ and $24 \mathrm{wk}$ comparisons are shown in Tables 5-8.

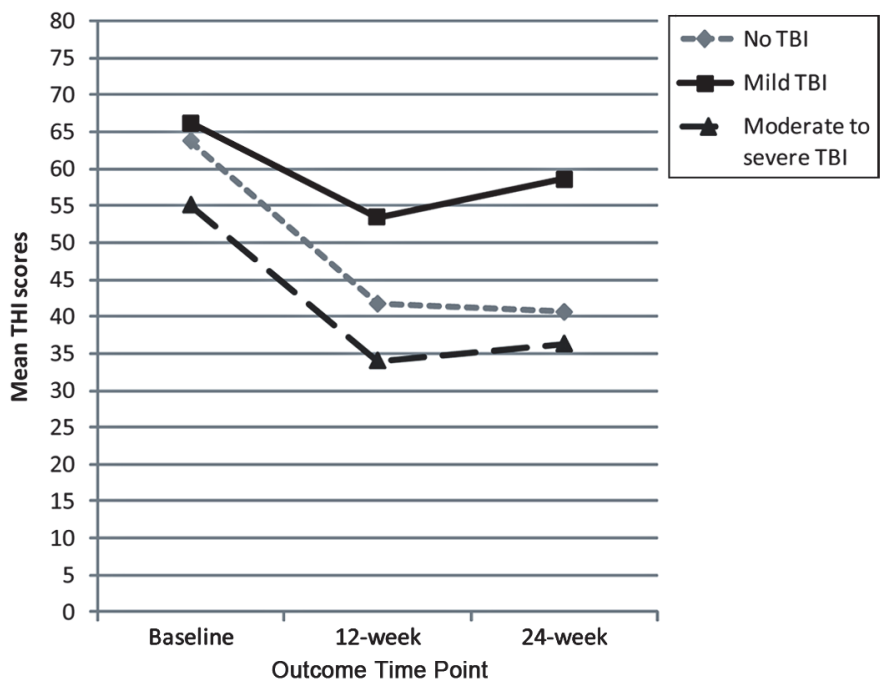

Figure 2.

Mean Tinnitus Handicap Inventory (THI) scores at baseline, 12week, and 24-week time points for each of three study groups. $\mathrm{TBI}=$ traumatic brain injury.

Table 4.

Numbers of participants who completed Tinnitus Handicap Inventory at each outcome point.

\begin{tabular}{lccc}
\hline \multicolumn{1}{c}{ Group } & Baseline & $\mathbf{1 2}$ wk & 24 wk \\
\hline No TBI & 12 & 8 & 11 \\
Mild TBI & 15 & 11 & 14 \\
Moderate-Severe TBI & 9 & 6 & 7 \\
Total & 36 & 25 & 32 \\
\hline TBI = traumatic brain injury. & \multicolumn{4}{l}{} \\
\hline \hline
\end{tabular}

Two participants (113 and 118) experienced large improvements in their THI scores that were outside the normal distribution. Because of the small sample size, the statistical tests were run both with and without these two participants. Although there were slight differences, for the most part, the results were similar. Overall, participants showed a significant reduction in mean THI scores from baseline to $12 \mathrm{wk}(p=0.002)$ and from baseline to $24 \mathrm{wk}(p<0.001)$. At $12 \mathrm{wk}$, only the mTBI group approached a significant reduction in mean THI scores $(p=0.01)$. At $24 \mathrm{wk}$, all three groups approached significant reductions in mean THI scores (noTBI: $p=0.01$, mTBI: $p=0.04$, m-sTBI: $p=0.02$ ). A Kruskal-Wallis test was performed to determine whether the mean reductions from baseline to $12 \mathrm{wk}$ and from baseline to $24 \mathrm{wk}$ differed between the three groups. No significant difference was detected $(p>0.05)$. 
JRRD, Volume 49, Number 7, 2012

Table 5.

Tinnitus Handicap Inventory (THI) scores (mean \pm standard deviation) and effect sizes by group, between baseline and 12 weeks.

\begin{tabular}{lrcccc}
\hline \multicolumn{1}{c}{ Group } & $\boldsymbol{n}^{*}$ & Baseline & 12 wk & Difference & Effect Size \\
\hline No TBI & 8 & $56.8 \pm 19.3$ & $41.8 \pm 28.8$ & $15.0 \pm 26.4$ & 0.57 \\
Mild TBI & 11 & $61.9 \pm 23.4$ & $53.4 \pm 28.3$ & 0.97 \\
Moderate to Severe TBI & 6 & $53.0 \pm 20.6$ & $34.0 \pm 24.5$ & $19.0 \pm 31.4$ \\
All Participants & 25 & $58.1 \pm 20.9$ & $45.0 \pm 27.7$ & $13.1 \pm 21.4$ & 0.60 \\
\hline
\end{tabular}

${ }^{*}$ Data for 25 total participants who completed THI at both time points.

$\mathrm{TBI}=$ traumatic brain injury.

Table 6.

Tinnitus Handicap Inventory (THI) scores (mean \pm standard deviation) and effect sizes by group, between baseline and 12 weeks-without outliers (participants 113 and 118).

\begin{tabular}{lrcccc}
\hline \multicolumn{1}{c}{ Group } & $\boldsymbol{n}^{*}$ & Baseline & 12 wk & Difference & Effect Size \\
\hline No TBI & 7 & $52.3 \pm 15.7$ & $44.6 \pm 29.9$ & $7.7 \pm 17.8$ & 0.43 \\
Mild TBI & 11 & $61.9 \pm 23.4$ & $53.4 \pm 28.3$ & $8.5 \pm 8.7$ & 0.97 \\
Moderate to Severe TBI & 5 & $48.0 \pm 18.5$ & $40.8 \pm 20.1$ & $7.2 \pm 13.8$ & 0.52 \\
All Participants & 23 & $55.9 \pm 20.4$ & $47.9 \pm 26.7$ & $7.9 \pm 12.4$ & 0.63 \\
\hline
\end{tabular}

*Data for 23 total participants who completed THI at both time points-excluding outliers (participants 113 and 118).

$\mathrm{TBI}=$ traumatic brain injury.

Table 7.

Tinnitus Handicap Inventory (THI) scores (mean \pm standard deviation) and effect sizes by group, between baseline and 24 weeks.

\begin{tabular}{lrcrrr}
\hline \multicolumn{1}{c}{ Group } & $\boldsymbol{n}^{*}$ & Baseline & 24 wk & Difference & Effect Size \\
\hline No TBI & 11 & $61.4 \pm 20.7$ & $40.7 \pm 26.9$ & $20.8 \pm 21.8$ & 0.95 \\
Mild TBI & 14 & $65.8 \pm 22.8$ & $58.6 \pm 28.9$ & 0.63 \\
Moderate to Severe TBI & 7 & $49.4 \pm 21.1$ & $36.3 \pm 25.6$ & $13.4 \pm 29.0$ & 0.45 \\
All Participants & 32 & $60.7 \pm 21.9$ & $47.5 \pm 28.5$ & $13.1 \pm 20.2$ & 0.65 \\
\hline
\end{tabular}

*Data for 32 total participants who completed THI at both time points.

$\mathrm{TBI}=$ traumatic brain injury.

Table 8.

Tinnitus Handicap Inventory (THI) scores (mean \pm standard deviation) and effect sizes by group, between baseline and 24 weeks - without outliers (participants 113 and 118).

\begin{tabular}{lrcccc}
\hline \multicolumn{1}{c}{ Group } & $\boldsymbol{n}^{*}$ & Baseline & 24 wk & Difference & Effect Size \\
\hline No TBI & 10 & $58.8 \pm 19.7$ & $43.0 \pm 27.3$ & $15.8 \pm 15.2$ & 1.03 \\
Mild TBI & 14 & $65.8 \pm 22.8$ & $58.6 \pm 28.9$ & 0.63 \\
Moderate to Severe TBI & 6 & $44.7 \pm 18.5$ & $42.3 \pm 21.9$ & $2.3 \pm 5.6$ & 0.41 \\
All Participants & 30 & $59.2 \pm 21.8$ & $50.1 \pm 27.5$ & $9.1 \pm 12.7$ & 0.72 \\
\hline
\end{tabular}

*Data for 30 total participants who completed THI at both time points—excluding outliers (participants 113 and 118).

$\mathrm{TBI}=$ traumatic brain injury.

\section{Effect Sizes}

Effect sizes indicate the magnitude of the "real" treatment effect, and their use standardizes outcome data across different instruments [45]. We calculated effect sizes for the THI outcomes at $12 \mathrm{wk}$ and $24 \mathrm{wk}$, with and without the two participant outliers, which are shown in Tables 5-8. Cohen suggests that, as a general rule of thumb, effect sizes of $0.20,0.50$, and 0.80 are considered small, medium, and large, respectively [46].
At $12 \mathrm{wk}$, the effect sizes for the noTBI group were 0.57 with outliers, 0.43 without outliers. The effect size for the mTBI group was 0.97 with and without outliers, and the effect sizes for the m-sTBI group were 0.60 with outliers, 0.52 without outliers. At $24 \mathrm{wk}$, the effect sizes for the noTBI group were 0.95 with outliers, 1.03 without outliers. The effect size for the mTBI group was 0.63 with and without outliers, and the effect sizes for the m-sTBI group were 0.45 with outliers and 0.41 without outliers. 


\section{Reflective Interviews}

Formative evaluation, in the form of reflective interviews, was conducted (by MWL) with the four study team members (research assistant, research coordinator, and two intervention counselors) who communicated with the research participants. The interviews were intended to elicit team members' observations and synthesize their informed suggestions for planning the next phase of the work [47]. These interviews occurred after all study participants were recruited and communication with participants was well underway. Notes were transcribed and reviewed by study team members, then combined and categorized by topics discussed.

The tinnitus materials and exercises in the pilot project were based on the PTM protocol [13]. Modifications were necessary to (1) deliver the PTM education by telephone, (2) include persons with various levels of TBI, and (3) include a psychologist for the first time. These modifications were explored with the study team members. A particular modification to the PTM protocol was to drop the requirement that participants had to first complete a hearing evaluation and be fitted with amplification when appropriate before receiving tinnitus counseling. Study team members recommended that this requirement be reinstituted in the follow-up study.

Some participants reported that their tinnitus distress resolved before completing all calls. However, participants were required to complete all telephone appointments with the intervention counselors rather than discontinue the intervention when they felt that they had received sufficient education. For these participants, the counselors continued to have short check-in telephone calls for the required number of appointments.

A number of comments were made by the study team members about providing these services by telephone rather than in a clinic. Before the pilot study, questions had been raised about whether this study population was appropriate for a telehealth approach. When queried, all four of the study team members reported that they did not experience difficulties hearing or being heard during calls. (Perhaps because the participants self-selected to participate in a study that involved care by telephone, all participants could communicate by telephone.) Participants reported to the study team members that they were glad to avoid travel for this care because of age, distance, disability, or work. In order to reduce the number of telephone appointments that were "no shows," reminder calls were placed with acceptable completion rates being achieved. Overall, the study team members found intervention offered by telephone to be a positive, feasible experience from their perspective and from the reports from the study participants.

The intervention counselors (audiologist and psychologist) indicated that use of a videophone might improve communicative interactions over the telephone. However, they were satisfied with communication by telephone alone and expressed concern that incorporating use of video technology would render long-distance communication too cumbersome and/or difficult to use for many people who might otherwise benefit from long-distance intervention by telephone alone.

The unmodified clinician materials (e.g., clinician guidelines handbook, Web-based online training of PTM) proved satisfactory to the study team members. Some additional training about TBI and PTSD was provided and deemed useful. Other materials, such as the self-help workbook from the PTM protocol, had undergone revision. It now included education about the use of CBT to be delivered by a psychologist. This was well received by participants and the intervention counselors were positive about continuing to develop and deliver the workbook. The inclusion of a third CBT skill, attention diversion via pleasant activities, will be used in the follow-up study.

The addition of a psychologist to the intervention team was strongly endorsed by all four study team members because of perceived benefit to the participants and other study team members. Although including a second clinician requires careful intervention planning between two departments, such collaboration is important because patients with clinically significant tinnitus (and patients with a history of TBI) often report comorbid depression and anxiety.

Following screening and recruitment, none of the participants was deemed by the study team members to be too impaired to benefit from a care plan. Often, the two intervention counselors were not aware of which TBI category their participant belonged to while receiving intervention. However, when an intervention counselor checked the status of a participant who was more impulsive or had difficulty keeping appointments and focusing on a task, that person was often, but not always, in the m-sTBI group.

The inclusion of a psychologist improved safety for a participant who expressed suicidal ideation, which is not an uncommon symptom of acute stress, anxiety, and depression [48]. When the audiologist was concerned about a patient, she would contact the psychologist and together they would determine what action needed to be taken. Many participants were provided the toll-free number for 
the National Suicide Prevention Hotline because of their elevated depressive symptoms.

Implementing this study required development of centralized databases to share schedules and intervention information. The intervention counselors were in two different geographic locations and time zones, and participants were located anywhere within the United States. All four study team members indicated that the centralized databases were essential to have common access to all relevant study data. They mentioned that in the next project they would like to have improved versions to provide seamless care. A separate issue that was reported was the sense that the two clinicians felt their work had not been very connected. "We worked on different goals with the patients. . . While we had a shared treatment database, we did not have a common treatment plan." One suggestion was to use the Tinnitus Problem Checklist [13] to identify issues both could address. (The Tinnitus Problem Checklist is the starting point for patients to develop self-management plans using the worksheets shown in Appendices 1 and 2.) This is an area that will receive attention during the follow-up study.

\section{DISCUSSION}

\section{Study Findings}

The purpose of this pilot study was to develop and test a prototype protocol for providing tinnitus-management services to Veterans who had experienced a TBI. The educational counseling that is used with PTM was enhanced with components of CBT, and the counseling was administered over the telephone to a limited number of participants located throughout the United States. Participants were grouped with respect to their TBI history: mTBI, m-sTBI, and noTBI. All three groups showed similar improvement in their mean THI scores, resulting in moderate to large effect sizes. These data, and the experiences gained from conducting this study, have been used to design a randomized clinical trial to more definitively evaluate the efficacy of this telehealth methodology. This 4-year clinical trial is underway.

It is noteworthy that certain differences appeared with respect to the participants' baseline tinnitus characteristics. For example, almost half the m-sTBI group reported that their tinnitus was perceived "inside the head," while only 13 and 8 percent of the mTBI and noTBI groups, respectively, reported this same perception. If this finding is repeated in the larger follow-up trial, then this could imply that tinnitus is categorically different for individuals who have experienced a major head injury. This kind of information could have implications regarding underlying mechanisms of tinnitus generation. Further, we previously conducted a randomized clinical study that included 269 participants [49]. They all were asked "what is the location of your tinnitus" and only 25 (9\% of 268 responses) reported that their tinnitus was located "inside the head." Almost all the remaining participants reported the perceived location of their tinnitus in one or both ears. This study group is typical of patients who complain of tinnitus, of which the majority report that their tinnitus was caused by noise exposure.

It was also noted that, compared with the noTBI group, both TBI groups (mTBI and m-sTBI) reported not getting enough sleep and a greater prevalence of anxiety and probable PTSD. These findings might be expected given the brain trauma experienced by these individuals. Most of the participants in this study screened negative for depression, although it was noted that the mTBI group screened positive for depression most often. These findings are consistent with the literature that provides substantial evidence that TBI is associated with sleep disturbance, anxiety, PTSD, and depression [50-55].

Based on this study's experiences with expressed suicidal ideation, we have revised our protocol to exclude any candidates on this basis. We now will require all candidates to undergo screening for suicidal ideation. The screening will be conducted by the psychologist as part of the initial assessment. If the candidate indicates current, active suicidal ideation, then the psychologist will contact local emergency responders to ensure safety [56]. The candidate will be considered a "screen failure" and excluded from study participation.

\section{Clinical Application}

The tinnitus management program that was developed for this study can be adopted by clinicians who are interested in implementing such a program. The program can be conducted by audiologists and mental health providers who have received the proper training. We are attempting to make PTM training available in a variety of formats (some of these materials can be provided upon request to the contact author):

1. PTM has been described in detail and the patient selfhelp workbook, clinician handbook, and counseling guide are available [13,57-58]. 
2. PTM live training seminars are conducted at various times and locations around the country.

3. Online PTM training has been developed that is available to VA clinicians and includes training modules that focus on patient education principles pertinent to tinnitus management and health literacy. (We hope to make this online training accessible to non-VA clinicians.)

4. DVDs of past PTM training seminars are available. (Please note that none of the authors can profit in any way from the sale or distribution of any of these training materials.)

The counseling used for this study was based on the information that is found in the PTM self-help workbook [58]. Clinicians can learn this counseling by carefully studying the workbook and by viewing the video presentations that are included in the workbook. These video presentations show the group counseling that is done for the two PTM workshops conducted by audiologists. Similar videos for the three PTM workshops that are conducted by psychologists are also available. When viewing the video presentations, clinicians are suggested to first play the role of the patient to gain the patient's perspective of the material that is taught. Clinicians can then practice using the PowerPoint presentations that have been developed for this purpose.

We cannot as yet make any definitive statements comparing the efficacy of performing the PTM counseling live in the clinic versus delivering it via telehealth. The methodology was developed fairly recently and studies are needed to determine its efficacy when conducted using different delivery modalities. Note that, increasingly, studies are showing the efficacy of using telehealth delivery of various mental health interventions [59-60]. A meta-analysis of 29 home-based telehealth studies revealed an overall positive effect on clinical outcomes in diverse patient populations [61]. The current follow-up study will more definitively assess outcomes of the PTM telehealth intervention.

\section{CONCLUSIONS}

Much of our research has focused on the development and validation of PTM, which can be implemented efficiently in VA hospitals. Our pilot study, described in the present article, showed positive results of conducting the PTM counseling via telephone. Adaptation of PTM as a home-based telehealth service has the potential of pro- viding needed tinnitus services to Veterans with and without TBI for a relatively small cost and with minimal effect on individual VA hospitals. The National Offices of Care Coordination and Audiology and Speech Pathology are vested in ensuring that providers consider telehealth options to expand the scope of specialized services that will improve access and to develop, adapt, and disseminate policies, best practices, lessons learned, and successful strategies to improve care. Upon conclusion of our follow-up study, the tinnitus telehealth program will be fully developed and organizational requirements and patient outcomes will be documented.

\section{ACKNOWLEDGMENTS}

\section{Author Contributions:}

Study concept and design: J. A. Henry, T. L. Zaugg, P. J. Myers, C. J. Schmidt, M. W. Legro, D. M. Storzbach, G. P. McMillan, K. F. Carlson.

Acquisition of data: T. L. Zaugg, C. J. Schmidt, S. Griest, C. Kaelin, E. J. Thielman.

Analysis and interpretation of data: S. Griest, E. J. Thielman, G. P. McMillan.

Drafting of manuscript: J. A. Henry, T. L. Zaugg, P. J. Myers, C. J. Schmidt, M. W. Legro, G. P. McMillan, K. F. Carlson.

Critical revision of manuscript for important intellectual content:

J. A. Henry, T. L. Zaugg, P. J. Myers, C. J. Schmidt, M. W. Legro, G. P. McMillan, K. F. Carlson.

Statistical analysis: S. Griest, E. J. Thielman, G. P. McMillan. Obtained funding: J. A. Henry, T. L. Zaugg, P. J. Myers.

Administrative, technical, or material support: C. Kaelin, E. J. Thielman. Study supervision: J. A. Henry, T. L. Zaugg, P. J. Myers.

Financial Disclosures: The authors have declared that no competing interests exist.

Funding/Support: This material was based on work supported by the VA, VHA, Rehabilitation Research and Development Service (grants C6770R, F7070S, C2659C). Dr. Carlson's efforts were supported by the VA, VHA, Health Services Research and Development Career Development Award Program (grant 08-025).

Additional Contributions: Our appreciation to Marjorie Leek, $\mathrm{PhD}$, Interim Director of the NCRAR, for her support of this project.

Institutional Review: All procedures for recruitment, informed consent, and conduct of this study adhered to the requirements of the IRBs at the PVAMC and VACHS for protection of human research participants.

Participant Follow-Up: The authors do not plan to inform participants of the publication of this study.

Disclaimer: The opinions expressed in this article are those of the authors and do not necessarily reflect the position or policy of the VA or the U.S. Government. 


\section{REFERENCES}

1. Lew HL, Poole JH, Guillory SB, Salerno RM, Leskin G, Sigford B. Persistent problems after traumatic brain injury: The need for long-term follow-up and coordinated care. J Rehabil Res Dev. 2006;43(2):vii-x. [PMID:16847779] http://dx.doi.org/10.1682/JRRD.2006.05.0054

2. Langlois JA, Rutland-Brown W, Thomas KE. Traumatic brain injury in the United States: emergency department visits, hospitalizations, and deaths. Atlanta (GA): Centers for Disease Control and Prevention, National Center for Injury Prevention and Control; 2006.

3. McCrea MA. Mild traumatic brain injury and postconcussion syndrome. The new evidence base for diagnosis and treatment. New York (NY): Oxford University Press; 2008.

4. Carroll LJ, Cassidy JD, Holm L, Kraus J, Coronado VG; WHO Collaborating Centre Task Force on Mild Traumatic Brain Injury. Methodological issues and research recommendations for mild traumatic brain injury: the WHO Collaborating Centre Task Force on Mild Traumatic Brain Injury. J Rehabil Med. 2004;43(Suppl):113-25. [PMID:15083875] http://dx.doi.org/10.1080/16501960410023877

5. Hoge CW, Goldberg HM, Castro CA. Care of war veterans with mild traumatic brain injury-flawed perspectives. N Engl J Med. 2009;360(16):1588-91. [PMID:19369664] http://dx.doi.org/10.1056/NEJMp0810606

6. Tanielian T, Jaycox LH, editors. Invisible wounds of war: psychological and cognitive injuries, their consequences, and services to assist recovery. Santa Monica (CA): RAND Center for Military Health and Policy; 2008.

7. Warden D. Military TBI during the Iraq and Afghanistan wars. J Head Trauma Rehabil. 2006;21(5):398-402. [PMID:16983225] http://dx.doi.org/10.1097/00001199-200609000-00004

8. Carlson KF, Nelson D, Orazem RJ, Nugent S, Cifu DX, Sayer NA. Psychiatric diagnoses among Iraq and Afghanistan war veterans screened for deployment-related traumatic brain injury. J Trauma Stress. 2010;23(1):17-24. [PMID:20127725]

9. Hoffman HJ, Reed GW. Epidemiology of tinnitus. In: Snow JB, editor. Tinnitus: theory and management. Lewiston (NY): BC Decker Inc; 2004. p. 16-41.

10. Cave KM, Cornish EM, Chandler DW. Blast injury of the ear: clinical update from the global war on terror. Mil Med. 2007;172(7):726-30. [PMID:17691685]

11. Lew HL, Jerger JF, Guillory SB, Henry JA. Auditory dysfunction in traumatic brain injury. J Rehabil Res Dev. 2007; 44(7):921-28. [PMID:18075949] http://dx.doi.org/10.1682/JRRD.2007.09.0140
12. Chandler DW, Edmond CV. Effects of blast overpressure on the ear: case reports. J Am Acad Audiol. 1997;8(2):81-88. [PMID:9101454]

13. Henry JA, Zaugg TL, Myers PM, Kendall CJ. Progressive tinnitus management: clinical handbook for audiologists. San Diego (CA): Plural Publishing; 2010.

14. Krumm M, Ferrari DV. Contemporary telehealth and telemedicine application in audiology. Audiol Today. 2008; 20(5):36-41.

15. Wakefield BJ. Telehealth. J Gerontol Nurs. 2001;27(1):9. [PMID:11915101]

16. Tuerk PW, Fortney J, Bosworth HB, Wakefield B, Ruggiero KJ, Acierno R, Frueh BC. Toward the development of national telehealth services: the role of Veterans Health Administration and future directions for research. Telemed $\mathbf{J}$ E Health. 2010;16(1):115-17. [PMID:20043704] http://dx.doi.org/10.1089/tmj.2009.0144

17. Hersh WR, Hickam DH, Severance SM, Dana TL, Krages KP, Helfand M. Telemedicine for the medicare population: update. Evidence report/technology assessment No. 131 (Prepared by the Oregon Evidence-Based Practice Center under contract No. 290-02-0024.) AHRQ Publication No. 06-E007. Rockville (MD): Agency for Healthcare Research and Quality; 2006.

18. Bell KR, Temkin NR, Esselman PC, Doctor JN, Bombardier CH, Fraser RT, Hoffman JM, Powell JM, Dikmen S. The effect of a scheduled telephone intervention on outcome after moderate to severe traumatic brain injury: a randomized trial. Arch Phys Med Rehabil. 2005;86(5):851-56. [PMID:15895327] http://dx.doi.org/10.1016/j.apmr.2004.09.015

19. Carlson KF, Kehle SM, Meis LA, Greer N, Macdonald R, Rutks I, Sayer NA, Dobscha SK, Wilt TJ. Prevalence, assessment, and treatment of mild traumatic brain injury and posttraumatic stress disorder: a systematic review of the evidence. J Head Trauma Rehabil. 2011;26(2):103-15. [PMID:20631631] http://dx.doi.org/10.1097/HTR.0b013e3181e50ef1

20. Martinez Devesa P, Waddell A, Perera R, Theodoulou M. Cognitive behavioural therapy for tinnitus. Cochrane Database Syst Rev. 2007;(1):CD005233. [PMID:17253549]

21. Soo C, Tate R. Psychological treatment for anxiety in people with traumatic brain injury. Cochrane Database Syst Rev. 2007;(3):CD005239. [PMID:17636792]

22. Henry JA, Zaugg TL, Myers PJ, Kendall CJ, Michaelides EM. A triage guide for tinnitus. J Fam Pract. 2010;59(7): 389-93. [PMID:20625568]

23. Henry JA, Zaugg TL, Myers PJ, Schechter MA. The role of audiologic evaluation in progressive audiologic tinnitus management. Trends Amplif. 2008;12(3):170-87.

[PMID:18628281]

http://dx.doi.org/10.1177/1084713808319941 
24. Henry JA, Zaugg TL, Myers PJ, Kendall CJ, Turbin MB. Principles and application of educational counseling used in progressive audiologic tinnitus management. Noise Health. 2009; 11(42):33-48. [PMID:19265252] http://dx.doi.org/10.4103/1463-1741.45311

25. Crocetti A, Forti S, Ambrosetti U, Bo LD. Questionnaires to evaluate anxiety and depressive levels in tinnitus patients. Otolaryngol Head Neck Surg. 2009;140(3):403-5. [PMID:19248952] http://dx.doi.org/10.1016/j.otohns.2008.11.036

26. Shargorodsky J, Curhan GC, Farwell WR. Prevalence and characteristics of tinnitus among US adults. Am J Med. 2010; 123(8):711-18. [PMID:20670725] http://dx.doi.org/10.1016/j.amjmed.2010.02.015

27. Zöger S, Svedlund J, Holgers KM. Psychiatric disorders in tinnitus patients without severe hearing impairment: 24 month follow-up of patients at an audiological clinic. Audiology. 2001;40(3):133-40. [PMID:11465295] http://dx.doi.org/10.3109/00206090109073108

28. Beck JS. Cognitive therapy: basics and beyond. New York (NY): Guilford; 1995.

29. Sweetow RW. Cognitive-behavior modification. In: Tyler RS, editor. Tinnitus handbook. San Diego (CA): Singular Publishing Group; 2000. p. 297-311.

30. Andersson G. Psychological aspects of tinnitus and the application of cognitive-behavioral therapy. Clin Psychol Rev. 2002;22(7):977-90. [PMID:12238249] http://dx.doi.org/10.1016/S0272-7358(01)00124-6

31. Henry JL, Wilson PH. Psychological treatments for tinnitus. In: Vernon JA, editor. Tinnitus treatment and relief. Needham Heights (MA): Allyn \& Bacon; 1998. p. 99-115.

32. Henry JA, Zaugg TL, Schechter MA, Myers PJ. How to manage your tinnitus: a step-by-step workbook. Portland (OR): VA National Center for Rehabilitative Auditory Research; 2008.

33. Myers P, Lorenz J, Weinel D. Written materials resource manual for patient health education. Tampa (FL): James A. Haley VA Patient Education Committee; 2003.

34. Henry JA, Zaugg TL, Myers PJ, Kendall CJ. How to manage your tinnitus: a step-by-step workbook. 2nd ed. Portland (OR): VA National Center for Rehabilitative Auditory Research; 2009.

35. Zaugg TL, Schechter MA, Fausti SA, Henry JA. Difficulties caused by patients' misconceptions that hearing problems are due to tinnitus. In: Patuzzi R, editor. Proceedings of Seventh International Tinnitus Seminar; 2002 Mar 5-9; Perth, Australia. Perth (Australia): Discipline of Physiology, School of Biomedical and Chemical Sciences, University of Western Australia; 2003. p. 226-28.

36. Iverson GL, Lange RT, Gaetz M, Zasler ND. Mild TBI. In: Zasler ND, Katz DI, Zafonte RD, editors. Brain injury medicine: principles and practice. New York (NY): Demos Medical Publishing; 2006. p. 333-71.

37. Zigmond AS, Snaith RP. The hospital anxiety and depression scale. Acta Psychiatr Scand. 1983;67(6):361-70. [PMID:6880820]

http://dx.doi.org/10.1111/j.1600-0447.1983.tb09716.x

38. Wilkinson MJ, Barczak P. Psychiatric screening in general practice: comparison of the general health questionnaire and the hospital anxiety depression scale. J R Coll Gen Pract. 1988;38(312):311-13. [PMID:3255827]

39. Newman CW, Jacobson GP, Spitzer JB. Development of the Tinnitus Handicap Inventory. Arch Otolaryngol Head Neck Surg. 1996;122(2):143-48. [PMID:8630207] http://dx.doi.org/10.1001/archotol.1996.01890140029007

40. Newman CW, Sandridge SA, Jacobson GP. Psychometric adequacy of the Tinnitus Handicap Inventory (THI) for evaluating treatment outcome. J Am Acad Audiol. 1998; 9(2):153-60. [PMID:9564679]

41. Newman CW, Sandridge SA. Tinnitus questionnaires. In: Snow JB, editor. Tinnitus: theory and management. Lewiston (NY): BC Decker Inc; 2004. p. 237-54.

42. Prins A, Ouimette P, Kimerling R, Cameron RP, Hugelshofer DS, Shaw-Hegwer J, Thrailkill A, Gusman FD, Sheikh JI. The primary care PTSD screen (PC-PTSD): development and operating characteristics. Prim Care Psychiatry. 2003; 9(1):9-14. http://dx.doi.org/10.1185/135525703125002360

43. Bliese PD, Wright KM, Adler AB, Cabrera O, Castro CA, Hoge CW. Validating the primary care posttraumatic stress disorder screen and the posttraumatic stress disorder checklist with soldiers returning from combat. J Consult Clin Psychol. 2008;76(2):272-81. [PMID:18377123] http://dx.doi.org/10.1037/0022-006X.76.2.272

44. Johns MW. A new method for measuring daytime sleepiness: the Epworth Sleepiness Scale. Sleep. 1991;14(6): 540-45. [PMID:1798888]

45. Lipsey MW. Design sensitivity: statistical power for experimental research. Newbury Park (CA): Sage; 1990.

46. Cohen J. Statistical power analysis for the behavioral sciences. 22nd ed. Hillsdale (NJ): Lawrence Erlbaum Associates, Inc; 1988.

47. Stetler CB, Legro MW, Wallace CM, Bowman C, Guihan M, Hagedorn H, Kimmel B, Sharp ND, Smith JL. The role of formative evaluation in implementation research and the QUERI experience. J Gen Intern Med. 2006;21(Suppl 2):S1-8. [PMID:16637954]

http://dx.doi.org/10.1007/s11606-006-0267-9

48. Borges G, Nock MK, Haro Abad JM, Hwang I, Sampson NA, Alonso J, Andrade LH, Angermeyer MC, Beautrais A, Bromet E, Bruffaerts R, de Girolamo G, Florescu S, Gureje O, Hu C, Karam EG, Kovess-Masfety V, Lee S, Levinson D, Medina-Mora ME, Ormel J, Posada-Villa J, Sagar R, Tomov T, Uda H, Williams DR, Kessler RC. Twelve-month 
prevalence of and risk factors for suicide attempts in the World Health Organization World Mental Health Surveys. J Clin Psychiatry. 2010;71(12):1617-28.

[PMID:20816034]

http://dx.doi.org/10.4088/JCP.08m04967blu

49. Henry JA, Loovis C, Montero M, Kaelin C, Anselmi KA, Coombs R, Hensley J, James KE. Randomized clinical trial: group counseling based on tinnitus retraining therapy. J Rehabil Res Dev. 2007;44(1):21-32. [PMID:17551855] http://dx.doi.org/10.1682/JRRD.2006.02.0018

50. Orff HJ, Ayalon L, Drummond SP. Traumatic brain injury and sleep disturbance: a review of current research. J Head Trauma Rehabil. 2009;24(3):155-65. [PMID:19461363] http://dx.doi.org/10.1097/HTR.0b013e3181a0b281

51. Ouellet MC, Savard J, Morin CM. Insomnia following traumatic brain injury: a review. Neurorehabil Neural Repair. 2004;18(4):187-98. [PMID:15669131] http://dx.doi.org/10.1177/1545968304271405

52. Fann JR, Hart T, Schomer KG. Treatment for depression after traumatic brain injury: a systematic review. J Neurotrauma. 2009;26(12):2383-2402. [PMID:19698070] http://dx.doi.org/10.1089/neu.2009.1091

53. Moldover JE, Goldberg KB, Prout MF. Depression after traumatic brain injury: a review of evidence for clinical heterogeneity. Neuropsychol Rev. 2004;14(3):143-54. [PMID:15673235] http://dx.doi.org/10.1023/B:NERV.0000048181.46159.61

54. Rosenfeld JV, Ford NL. Bomb blast, mild traumatic brain injury and psychiatric morbidity: a review. Injury. 2010; 41(5):437-43. [PMID:20189170] http://dx.doi.org/10.1016/j.injury.2009.11.018

55. Halbauer JD, Ashford JW, Zeitzer JM, Adamson MM, Lew HL, Yesavage JA. Neuropsychiatric diagnosis and management of chronic sequelae of war-related mild to moderate traumatic brain injury. J Rehabil Res Dev. 2009;46(6):757-96. [PMID:20104402] http://dx.doi.org/10.1682/JRRD.2008.08.0119

56. Godleski L, Nieves JE, Darkins A, Lehmann L. VA telemental health: suicide assessment. Behav Sci Law. 2008;
26(3):271-86. [PMID:18548515]

http://dx.doi.org/10.1002/bsl.811

57. Henry JA, Zaugg TL, Myers PM, Kendall CJ. Progressive tinnitus management: counseling guide. San Diego (CA): Plural Publishing; 2010.

58. Henry JA, Zaugg TL, Myers PM, Kendall CJ. How to manage your tinnitus: a step-by-step workbook. 3rd ed. San Diego (CA): Plural Publishing; 2010.

59. Simon GE, Ludman EJ, Rutter CM. Incremental benefit and cost of telephone care management and telephone psychotherapy for depression in primary care. Arch Gen Psychiatry. 2009;66(10):1081-89. [PMID:19805698] http://dx.doi.org/10.1001/archgenpsychiatry.2009.123

60. Tutty S, Ludman EJ, Simon G. Feasibility and acceptability of a telephone psychotherapy program for depressed adults treated in primary care. Gen Hosp Psychiatry. 2005;27(6): 400-410. [PMID:16271654] http://dx.doi.org/10.1016/j.genhosppsych.2005.06.009

61. Dellifraine JL, Dansky KH. Home-based telehealth: a review and meta-analysis. J Telemed Telecare. 2008;14(2): 62-66. [PMID:18348749] http://dx.doi.org/10.1258/jtt.2007.070709

Submitted for publication July 3, 2010. Accepted in revised form May 16, 2011.

This article and any supplementary material should be cited as follows:

Henry JA, Zaugg TL, Myers PJ, Schmidt CJ, Griest S, Legro MW, Kaelin C, Thielman EJ, Storzbach DM, McMillan GP, Carlson KF. Pilot study to develop telehealth tinnitus management for persons with and without traumatic brain injury. J Rehabil Res Dev. 2012;49(7): $1025-42$.

http://dx.doi.org/10.1682/JRRD.2010.07.0125

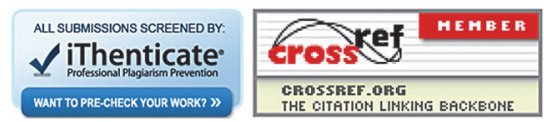

